\title{
Correction to: SQL-Like Interpretable Interactive Video Search
}

Jiaxin Wu, Phuong Anh Nguyen, Zhixin Ma, and Chong-Wah Ngo

\section{Correction to: \\ Chapter "SQL-Like Interpretable Interactive Video Search" in: J. Lokoč et al. (Eds.): MultiMedia Modeling, LNCS 12573, https://doi.org/10.1007/978-3-030-67835-7_34}

The original version of the book was inadvertently published with an incorrect acknowledgement in chapter 34 . The acknowledgement has been corrected and reads as follows:

Acknowledgement: The research was partially supported by the Singapore Ministry of Education (MOE) Academic Research Fund (AcRF) Tier 1 grant and the National Natural Science Foundation of China (No. 61872256).

The affiliation of the third author, Zhixin Ma, was incorrect. In the contribution it read "School of Information System," but correctly it should be "School of Computing and Information Systems".

The affiliation of the last author, Chong-Wah Ngo, was not correct. In the book it read "Department of Computer Science, City University of Hong Kong, Hong Kong, China". Instead, the correct affiliation is: "School of Computing and Information Systems, Singapore Management University, Singapore, Singapore”.

Additionally, his e-mail address "cscwngo@cityu.edu.hk" was also incorrect. The correct e-mail address is: "cwngo@smu.edu.sg".

The chapter and the book have been updated with the changes.

The updated version of this chapter can be found at https://doi.org/10.1007/978-3-030-67835-7_34 